Commentary

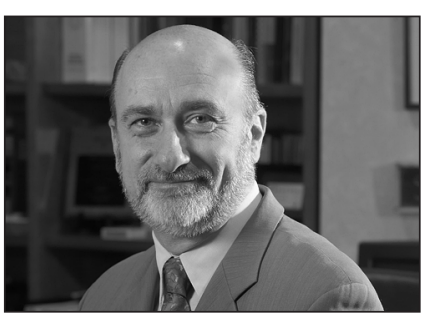

William K. Evans, MD, FRCPC Bill Evans, MD, $\operatorname{FRCP}(C)$, is a medical oncologist and President of the Juravinski Cancer Centre (JCC) at Hamilton Health Sciences and a Professor in the Department of Oncology at McMaster University. For the past 12 years, he has chaired the Provincial Lung Disease Group developing practice guidelines through Cancer Care Ontario's Program in Evidence-Based Care.

Melissa C. Brouwers, PhD Melissa C. Brouwers, PhD, is the Director of the Program in EvidenceBased Care, Cancer Care Ontario and an Associate Professor (PT) in the Department of Clinical Epidemiology and Biostatistics, McMaster University. She holds a BSc in Psychology from the University of Toronto and an MA and $\mathrm{PhD}$ in Psychology from the University of Western Ontario. She is an active and leading member of various national and international research groups including Clinical Practices Guidelines Action Group of the Canadian Strategy for Cancer Control, AGREE Next Steps, and ADAPTE.

Chaim M. Bell, MD, PhD, FRCPC

Chaim Bell, MD, PhD, $\operatorname{FRCP}(C)$, is a general internist and health services and health policy researcher at St Michael's Hospital in Toronto. His research in drug and technology policy focuses on cost-effectiveness. He is a member of both the National Joint Oncology Drug Review Committee for Canada and the Ontario Health Technology Advisory Committee that decides upon new oncology drug and technology funding.

The ideas and viewpoints expressed in this commentary are those of the authors and do not necessarily represent any policy, position, or program of the NCCN.
Journal of the National Comprehensive Cancer Network

\section{Should Cost of Care be Considered in a Clinical Practice Guideline?}

The global increase in cancer has fueled a large investment into research for more effective treatment strategies. The payoff from this investment has been a knowledge explosion that is overwhelming to the average oncologist. In response, a knowledge-synthesis industry has developed that uses the tools of systematic searches and standardized analytic processes to make sense of the large volume of frequently contradictory literature. Experts review the evidence and provide interpretation in the context of their professional values to guide their colleagues in clinical practice. Typically, guideline developers focus on questions of clinical effectiveness and efficacy, but rarely, if ever, consider the budgetary impacts of implementing the guideline or the cost-effectiveness of a new treatment. This issue of JNCCN focuses on guidelines for small cell and non-small cell lung cancer produced by the NCCN; neither of these guidelines mentions cost. In this commentary, we look at guideline development processes in general and specifically in Ontario, Canada, to consider the question: Is this an important omission or an irrelevancy?

For more than a decade, a multidisciplinary Lung Disease Site Group (LDSG) in the province of Ontario, Canada, has developed practice guidelines through Cancer Care Ontario's Program in Evidence-Based Care (PEBC), based at McMaster University. The LDSG has produced 25 clinical practice guidelines (CPGs), including 12 for non-small cell lung cancer and 4 for small cell lung cancer. Guidelines were also developed for each of the 8 new chemotherapeutic agents introduced since 1995 for the treatment of lung cancer, the radiotherapeutic management of lung cancer ( 10 guidelines), and the management of less common intrathoracic tumours (mesothelioma, thymoma). Guideline development has followed the practice guideline development cycle described by Browman et al. ${ }^{1}$

Guideline development begins with a systematic review of the Englishlanguage literature with a focus on randomized clinical trials. The PEBC search strategy purposefully does not include a search for articles on cost or cost-effectiveness. Although a consideration of the cost-effectiveness of new interventions is not part of the formal process of guideline development, members of the LDSG sometimes raise concerns that expensive drugs may not be funded through the provincial drug funding mechanism.

The draft guideline goes through a structured process of external review whereby clinicians involved in providing care to patients with lung cancer ensure that no relevant data were overlooked, provide comment on the level of support for the recommendations, and assist in knowledge transfer. ${ }^{2,3}$ This process asks practitioners to consider barriers to guideline implementation, and cost of the intervention is often a reported concern. The final guideline is submitted as a manuscript for publication and posted on Cancer Care Ontario's Web site (www.cancercare.on.ca).

The original intent of the CPGs was to develop a convenient source of highquality information to guide clinicians providing care to patients. However, soon after the program was established, the guidelines became the key clinical data summary for those involved in making funding decisions about new anticancer drugs 
for the provincial publicly financed health care system. ${ }^{4}$ However, the guidelines do not indicate whether the new therapy provides good value for money. So why is information on drug cost, economic impact, cost-effectiveness, or cost-utility not included in the guideline? There are a few good and some not so good reasons for this.

The first reason is historical. Although the Ontario Ministry of Health was among the first jurisdictions to require pharmaceutical manufacturers to submit data on the cost-effectiveness of their oral therapeutic products, Cancer Care Ontario, which had responsibility for the review of intravenous cancer drugs, did not require economic data for its decision-making process. Furthermore, the leadership of the PEBC and most members of the disease site groups (DSGs) strongly resisted the idea of incorporating cost information into guidelines.

Their reasons were threefold. From a methodological perspective, they cited concerns about the value and generalizabilty of economic data from other jurisdictions to decision-making in Ontario. In addition, the DSGs did not typically include members with expertise in the science and application of pharmacoeconomics, system resource allocation, or policy. The DSGs believed that the guideline development process should capitalize on the clinical expertise of oncologists, who constituted most of the membership, and focus deliberations on defining the clinical benefit. Finally, experts generally believed that clinicians were primarily responsible for advocating on behalf of their patients when evidence showed that a drug or therapy had the potential for clinical benefit. It was felt that the onus for obtaining economic data should be placed on the policy maker, not the clinician. These are the principal reasons why Cancer Care Ontario guideline development groups do not attempt to provide information on drug costs or cost-effectiveness in their guideline products.

A consolidated process is now established to review all new anticancer drugs (oral and intravenous) before funding approval can be obtained in Ontario. This process requires that economic data, which are provided by the pharmaceutical industry, be included in the decisions about drug approval. An obvious strength of having decision makers informed by CPGs prepared through the $\mathrm{PEBC}$ is that the guidelines are seen as the product of a legitimate process, independent of industry involvement. Thus, if cost-effectiveness information is now critical to the decision-making process, why is it not part of the guideline development process or even a parallel process? Surely asking industry to prepare the pharmacoeconomic analysis has the potential to introduce bias and undermine the decision-making process.

Several reasons explain why this is the current situation. Health economists are in relatively short supply, and those working in academic institutions are interested in advancing knowledge of health economics across a broad spectrum of activity and are not particularly enthusiastic about undertaking a large number of pharmacoeconomic analyses of cancer drug trials.

A second reason is that most clinical trials do not collect much, if any, resource utilization data, and health economists who are asked to undertake an economic analysis based on a clinical trial result are challenged to build models of care and outcomes informed by assumptions of event frequency, resource use, and unit costs that may or may not be relevant to the requesting jurisdiction. ${ }^{5}$ This is particularly challenging when a trial has been undertaken in multiple countries where patterns of practice and resource use may be quite different. ${ }^{6}$ A clinical trial with a pharmacoeconomic analysis conducted in a European country may be of little relevance to clinicians in Canada or the United States because of practice pattern differences. ${ }^{5,6}$

A third challenge is that the timeframe of data collection for most clinical trials is too short to gather the information needed to perform an ideal economic analysis. Data collection usually stops when the clinical end point of the trial is reached. Therefore, data on the late effects of therapy or subsequent treatments and the resources used to provide this care are incomplete.

Moreover, although many trials capture information on quality of life, they rarely capture standardized and economically grounded measures of patient preference called utilities. ${ }^{7}$ Utilities enable the economic analysis to be presented as a cost per quality-adjusted life-year. This allows for comparison of interventions, because the quality of the survival gain, rather than just the amount, is incorporated into the analysis. Ideally, utilities would be captured during clinical trials, but this becomes "just one more thing" to do in the already burdensome task of data collection for clinical trials; thus, utilities are not generally captured. Finally, even if good pharmacoeconomic analyses are 
conducted by industry, they would still be suspect because of the perceived conflict of interest.

If pharmacoeconomic analyses are important to decision makers (and they certainly are in Canada and other jurisdictions worldwide), then what are the implications for the future? In our view, the changes needed have less to do with guideline development groups than with the need for new approaches to clinical trials and new infrastructure to support decision makers. Going forward, we see little choice but for clinical trials groups and industry to collect resource utilization data during randomized trials.

The National Cancer Institute of Canada (NCIC) established a Working Group in Economic Analysis a decade ago that has made recommendations on which trials conducted by the NCIC Clinical Trials Group should have pharmacoeconomic analyses conducted concurrently. ${ }^{7}$ Some studies are now coming to fruition and are enabling the performance of highquality economic analyses.

A need also exists to collect utility values using tools such as the EQ5D or Health Utility Index. ${ }^{8}$ Research into the correlation between quality-of-life instruments and utilities would also be helpful, because it might inform how the vast quantity of quality-of-life data collected in clinical trials could be integrated into economic analyses in the future. In addition, new methodologies are needed that can facilitate the explicit incorporation of social and political values into health care rationing decisions.

Because little pharmacoeconomic research activity is associated with most of the major cooperative clinical trials groups, pharmacoeconomic units must be established to undertake independent evaluations of the cost-effectiveness of anticancer drugs. The British Columbia Cancer Agency has already established such a unit, and Ontario is in the planning stage. The independence of these analytic units creates a firewall between the guideline development process and industry and would eliminate the potential for systematic bias.

Decision makers with access to high-quality clinical evidence from guideline development groups and high-quality pharmacoeconomic analyses from independent credible health economics units would be better armed to make the difficult decisions that are increasingly being asked of them. Although this type of decision making will never be an easy task, incorporating rigorous cost-effectiveness analyses into the process would provide health care payors with greater confidence that they are funding therapies that provide good value for money. It would also assure those affected by lung cancer that they are being treated equitably in relation to those with other cancers.

\section{References}

1. Browman GP, Levine MN, Mohide EA, et al. The practice guideline development cycle: a conceptual tool for practice guidelines development and implementation. J Clin Oncol 1995;13:502-512.

2. Brouwers MC, Graham ID, Hanna SE, et al. Clinician's assessments of practice guidelines in oncology: the CAPGO survey. Int J Technol Assess Health Care 2004;20:421-426.

3. Browman GP, Newman TE, Mohide EA, et al. Progress of clinical oncology guidelines development using the practice guidelines development by cycle: the role of practitioner feedback. J Clin Oncol 1998;16:1226-1231.

4. Evans WK, Nefsky M, Pater J, et al. Cancer Care Ontario's New Drug Funding Program: controlled introduction of expensive anticancer drugs. Chronic Dis Can 2002;23:152-156.

5. Reed S, Anstrom K, Bakhai A, et al. Conducting economic evaluations alongside multinational clinical trials: toward a research consensus. Am Heart J 2005;149:434-443.

6. van Erkel AR, van den Hout WB, Pattynama PM. International differences in health care costs in Europe and the United States: do these affect the cost-effectiveness of diagnostic strategies for pulmonary embolism? Eur Radiol 1999;9:1926-1931.

7. Drummond M. Introducing economic and quality of life measurements into clinical studies. Ann Med 2001;33:344-349.

8. Evans WK, Coyle D, Gafni A, et al. Which cancer clinical trials should be considered for economic evaluation? Selection criteria from the National Cancer Institute of Canada's Working Group on Economic Analysis. Chronic Dis Can 2003;24:102-107. 